\title{
A RANDOM VERSION OF SCHAEFER'S FIXED POINT THEOREM WITH APPLICATIONS TO FUNCTIONAL RANDOM INTEGRAL EQUATIONS
}

\author{
B. C. DHAGE
}

\begin{abstract}
In this paper a random version of a fixed-point theorem of Schaefer is obtained and it is further applied to a certain nonlinear functional random integral equation for proving the existence result under Caratheodory conditions.
\end{abstract}

\section{Introduction}

Let $(\Omega, \mathcal{A})$ be a measurable space and let $X$ be a Banach space with a Borel $\sigma$-algebra $\beta_{X}$. A mapping $x: \Omega \times X \rightarrow X$ is called random variable if for a $B \in \beta_{X^{\prime}}, x^{-1}(B) \in \mathcal{A}$. A mapping $T: \Omega \times X \rightarrow X$ is called random operator if $T(., x)$ is measurable for each $x \in X$, and is generally expressed as $T(\omega, x):=T(\omega) x$. A random variable $\xi: \Omega \rightarrow X$ is called random fixed point of the random operator $T(\omega): \Omega \times X \rightarrow X$ if $T(\omega) \xi(\omega)=\xi(\omega)$ for each $\omega \in \Omega$. A random operator $T: \Omega \times X \rightarrow X$ is called continuous if $T(\omega)($.$) is$ continuous for each $\omega \in \Omega, T(\omega)$ is called totally bounded if for any bounded set $B$ in $X$, $T(\omega)(B)$ is a totally bounded subset of $X$ for each $\omega \in \Omega$. Similarly a random operator $T(\omega)$ is called completely continuous on $X$ if it is continuous and totally bounded random operator on $X$. Again the random operator $T: \Omega \times X \rightarrow X$ is called compact if $\overline{T(\omega)(X)}$ is a compact subset of $X$ for each $\omega \in \Omega$. Note that every compact random operator is totally bounded, but the reverse implication may not hold. However, two notions are equivalent on a bounded subset of a Banach space $X$. Finally the random operator $T: \Omega \times X \rightarrow X$ is called contraction if for each $\omega \in \Omega$,

$$
\|T(\omega) x-T(\omega) y\| \leq k(\omega)\|x-y\|
$$

for all $x, y \in X$, where $0 \leq k(\omega)<1$.

A Kuratowskii measure $\alpha$ of noncompactness of a bounded set $A$ in $X$ is a nonnegative real number $\alpha(A)$ defined by

$$
\alpha(A)=\inf \left\{r>0: A=\bigcup_{i=1}^{n} A_{i} ; \operatorname{diam}\left(A_{i}\right) \leq r, \forall i\right\} .
$$

Received December 4, 2002; revised February 10, 2003.

2000 Mathematics Subject Classification. 47H10.

Key words and phrases. Random fixed point theorem, random integral equation. 
A random operator $T(\omega)$ is called $\alpha$-condensing if for any bounded set $A$ in $X$, $T(\omega)(A)$ is bounded and $\alpha(T(\omega)(A))<\alpha(A)$ if $\alpha(A)>0$, for each $\omega \in \Omega$.

It is known that contraction and compact random operators are $\alpha$-condensing but the converse may not be true. We shall obtain a random version of the following generalization of Schaefer's nonlinear alternative due to Martelli [9].

Theorem A. [10] Let $X$ be a Banach space and let $T: X \rightarrow X$ be a continuous and $\alpha$-condensing map. Then either

(a) $T$ has a fixed point, or

(b) the set $\mathcal{E}=\{x \in X \mid x=\lambda T x, \lambda \in(0,1)\}$ is unbounded.

\section{Random Fixed Point Theory}

Theorem 2.1. Let $X$ be a separable Banach space and let $T: \Omega \times X \rightarrow X$ be a random operator satisfying for each $\omega \in \Omega$,

(a) $T(\omega)$ is continuous, and $\alpha$-condensing, and

(b) the set $\mathcal{E}=\{x \in X \mid x=\lambda(\omega) T(\omega) x\}$ is bounded for any measurable function $\lambda: \Omega \rightarrow \boldsymbol{R}$ with $0<\lambda(\omega)<1$.

Then $T(\omega)$ has a random fixed point.

Proof. Let $\omega \in \Omega$ be fixed. Then by a theorem of Martelli [9], $T(\omega)$ has a fixed point. We denote

$$
F(\omega)=\{x \in X \mid T(\omega) x=x\} .
$$

Obviously $F(\omega)$ is non-empty and compact for each $\omega \in \Omega$, since $T(\omega)$ is $\alpha$-condensing on $X$. To finish, it is enough to prove that the set-map $F: \Omega \rightarrow K(X)$ is measurable and has closed values. Let $C$ be a closed subset of $X$ and let $\left\{x_{n}\right\}$ be a dense subset of C. Define

$$
L(C)=\bigcap_{n=1}^{\infty} \bigcup_{x_{i} \in C_{n}}\left\{\omega \in \Omega \mid\left\|x_{i}-\omega x_{i}\right\|<\frac{1}{n}\right\}
$$

for all $C_{n}=\left\{x \in X \mid d(x, C)<\frac{1}{n}\right\}$, where $d(x, C)=\inf \{d(x, c) \mid c \in C\}$.

Clearly $L(C) \in \mathcal{A}$. Now it is shown as in Itoh [7] that $L(C)=F^{-1}(C)$. Hence $F(\omega)$ is measurable. Let $\left\{x_{n}\right\}$ be a sequence in $F(\omega)$ such that $x_{n} \rightarrow x$. Since $T(\omega)$ is continuous, $x_{n}=T(\omega) x_{n}$ implies $x=T(\omega) x$ which yields that $x \in F(\omega)$. Hence $F(\omega)$ is closed for each $\omega \in \Omega$, i.e. the set-map $F: \Omega \rightarrow K(X)$ has closed values. Now by an application of a selection theorem of Kuratowskii and Nardzewaski [8], the set-valued map $F$ has a measurable selection $\xi: \Omega \rightarrow X$ such that $\xi(\omega) \in F(\omega)$ for all $\omega \in \Omega$. This completes the proof.

Corollary 2.1. Let $X$ be a separable Banach space and let $T: \Omega \times X \rightarrow X$ be a random operator satisfying for each $\omega \in \Omega$,

(a) $T(\omega)$ is completely continuous,

(b) the set $\mathcal{E}=\{x \in X \mid T(\omega) x=\alpha(\omega) x\}$ is bounded, for any measurable function $\alpha$ :

$\Omega \rightarrow \boldsymbol{R}^{+}$with $\alpha(\omega)>1$. 
Then $T(\omega)$ has a random fixed point.

Before proving the next fixed point result, we give a useful definition.

Definition 2.1. A random operator $T: \Omega \times X \rightarrow X$ is called D-Lipschitzician if there exists a continuous nondecreasing function $\phi: \Omega \times \boldsymbol{R}^{+} \rightarrow \boldsymbol{R}^{+}$satisfying for each $\omega \in \Omega$,

$$
\|T(\omega) x-T(\omega) y\| \leq \phi_{\omega}(\|x-y\|)
$$

for all $x, y \in X$, where $\phi_{\omega}(r)=\phi_{\omega}(\omega, r)$ with $\phi(\omega, 0)=0$. The special case when $\phi_{\omega}(r)=\alpha(\omega) r, \alpha(\omega)>0$ for all $\omega \in \Omega, T(\omega)$ is called Lipschtzician with Lipschitz constant $\alpha(\omega), \omega \in \Omega$. In particular if $\alpha(\omega)<1$ for all $\omega \in \Omega$, then $T(\omega)$ is called a contraction with contraction constant $\alpha(\omega)$. Again if $\phi_{\omega}(r)<r, r>0$ for each $\omega \in \Omega$, then $T(\omega)$ is called a nonlinear contraction.

Our next results are random versions of the fixed point result of Krasnoselskii [6] and Dhage [3] in the framework of Schaefer fixed point theorem [10].

Theorem 3.2. Let $S(\omega), T(\omega): X \rightarrow X, X$ a separable Banach space, be two random operators satisfying for each $\omega \in \Omega$,

(a) $s(\omega)$ is nonlinear contraction,

(b) $T(\omega)$ is completely continuous, and

(c) the set $\mathcal{E}=\{x \in X \mid S(\omega) x+T(\omega) x=\alpha(\omega) x\}$ is bounded, for any measurable function $\alpha: \Omega \rightarrow \boldsymbol{R}^{+}$with $\alpha(\omega)>1$.

Then the random equation

$$
S(\omega) x+T(\omega) x=x
$$

has a random solution.

Proof. Define a random operator $Q(\omega): \Omega \times X \rightarrow X$ by

$$
Q(\omega) x=S(\omega) x+T(\omega) x .
$$

Obviously $Q(\omega)$ is a continuous random operator on $X$. Now for any $x, y \in X$, one has

$$
\begin{aligned}
\|Q(\omega) x-Q(\omega) y\| & \leq\|S(\omega) x-S(\omega) y\|+\|T(\omega) x-T(\omega) y\| \\
& \leq \phi_{\omega}(\|x-y\|)+\|T(\omega) x-T(\omega) y\| .
\end{aligned}
$$

Then for a fixed $\omega \in \Omega$, we have from (2.6),

$$
\alpha(T(\omega))(B) \leq \phi_{\omega}(\alpha(B))<\alpha(B) \quad \text { if } \alpha(B)>0,
$$

for a bounded subset $B$ of $X$, and so $T(\omega)$ is a $\alpha$-condensing random operator on $X$. Now an application of Theorem 2.1 yields that $T(\omega)$ has a random fixed point and consequently the random equation (2.4) has a random solution. The proof is complete.

Corollary 2.2. Let $X$ be a separable Banach space and let $S, T: Q \times X \rightarrow X$ be two random operators satisfying for each $\omega \in \Omega$, 
(a) $S(\omega)$ is contraction,

(b) $T(\omega)$ is completely continuous, and

(c) the set $\xi=\{x \in X \mid S(\omega) x+T(\omega) x=\alpha(\omega) x\}$ is bounded, for any measurable $\alpha: \Omega \rightarrow \boldsymbol{R}^{+}$with $\alpha(\omega)>1$.

Then the random equation (2.4) has a random solution.

Theorem 2.3. Let $X$ a separable Banach algebra and let $S, T: \Omega \times X \rightarrow X$ be two random operators satisfying for each $\omega \in \Omega$,

(a) $s(\omega)$ is D-Lipschitzician,

(b) $T(\omega)$ is continuous and compact and

(c) the set $\mathcal{E}=\{x \in X \mid S(\omega) x T(\omega) x=\alpha(\omega) x\}$ is bounded, for any measurable $\alpha: \Omega \rightarrow$ $\boldsymbol{R}^{+}$with $\alpha(\omega)>1$.

Then the random equation

$$
S(\omega) x T(\omega) x=x
$$

has a random solution whenever $M(\omega) \phi_{\omega}(r)<r, r>0$ for each $\omega \in \Omega$, where

$$
M(\omega)=\|T(\omega)(X)\|=\sup \{\|x(\omega)\|: x \in T(\omega)(X)\} .
$$

Proof. Let $\omega \in \Omega$ be fixed and define a mapping $Q: \Omega \times X \rightarrow X$ by

$$
Q(\omega)=S(\omega) x T(\omega) x
$$

Clearly $Q()$.$x is measurable for each x \in X$, and hence $Q(\omega)$ is a random operator. Let $B$ be a bounded subset of $X$ and let $x, y \in B$ be any two points. Then by (2.8),

$$
\begin{aligned}
\|Q(\omega) x-Q(\omega) y\| & =\|S(\omega) x T(\omega) x-S(\omega) y T(\omega) y\| \\
& \leq\|T(\omega) x\|\|S(\omega) x-S(\omega) y\|+\|S(\omega) x\|\|T(\omega) x-T(\omega) y\| \\
& \leq\|T(\omega)(B)\|\|S(\omega) x-S(\omega) y\|+\|S(\omega)(B)\|\|T(\omega) x-T(\omega) y\| \\
& \leq\|T(\omega)(X)\| \phi_{\omega}(\|x-y\|)+\|S(\omega)(B)\|\|T(\omega) x-T(\omega) y\|
\end{aligned}
$$

Now for a fixed $x_{0} \in B$,

$$
\begin{aligned}
\|S(\omega) x\| & \leq\left\|S(\omega) x_{0}\right\|+\left\|S(\omega) x-S(\omega) x_{0}\right\| \\
& \leq\left\|S(\omega) x_{0}\right\|+\phi_{\omega}\left(\left\|x-x_{0}\right\|\right) \\
& \leq\left\|S(\omega) x_{0}\right\|+\phi_{\omega}(\operatorname{diam} B)
\end{aligned}
$$

and therefore,

$$
\begin{aligned}
\|S(\omega)(B)\| & =\sup \{\|S(\omega) x\|: x \in B\} \\
& \leq\left\|S(\omega) x_{0}\right\|+\phi_{\omega}(\operatorname{diam} B) \\
& <\infty .
\end{aligned}
$$


Hence from (2.9) it follows that

$$
\|Q(\omega) x-Q(\omega) y\| \leq M(\omega) \phi_{\omega}(\|x-y\|)+\beta(\omega)\|T(\omega) x-T(\omega) y\|
$$

for all $x, y \in B$, where $\beta(\omega)=\left\|S(\omega) x_{0}\right\|+\phi_{\omega}(\operatorname{diam} B)<\infty$.

Now proceeding as in Dhage [3], it is proved that

$$
\alpha(Q(\omega)(B)) \leq M(\omega) \phi_{\omega}(\alpha(B))<\alpha(B), \quad \alpha(B)>0 .
$$

This shows that $Q(\omega)$ is a $\alpha$-condensing random operator on $X$. To prove the continuity of $Q(\omega)$, let $\left\{x_{n}\right\}$ be a sequence is $X$ converging to point $x$ in $X$. Then we have

$$
\begin{aligned}
\left\|Q(\omega) x_{n}-Q(\omega) x\right\| & \leq\left\|T(\omega) x_{n}\right\|\left\|S(\omega) x_{n}-S(\omega) x\right\|+\|S(\omega) x\|\left\|T(\omega) x_{n}-T(\omega) x\right\| \\
& \leq M(\omega) \phi_{\omega}\left(\left\|x_{n}-x\right\|\right)+\|S(\omega) x\|\left\|T(\omega) x_{n}-T(\omega) x\right\| \\
& \rightarrow 0 \text { as } n \rightarrow \infty
\end{aligned}
$$

which shows that $Q(\omega)$ is a continuous random operator on $X$. Now an application of Theorem 2.1 yields that $Q(\omega)$ has a random fixed point and consequently the random equation (2.7) has a random solution. This completes the proof.

Corollary 2.3. Let $X$ be a separable Banach algebra and let $S, T: \Omega \times X \rightarrow X$ be two random operators satisfying for each $\omega \in \Omega$,

(a) $S(\omega)$ is Lipschitzician with Lipschitz constant $\alpha(\omega)$,

(b) $T(\omega)$ is continuous and compact, and

(c) the set $\mathcal{E}=\{x \in X \mid S(\omega) x T(\omega) x=\lambda(\omega) x\}$ is bounded, for any measurable $\lambda: \Omega \rightarrow$ $\boldsymbol{R}^{+}$with $\lambda(\omega)>1$.

Then the operator equation (2.8) has a random solution whenever $\alpha(\omega) M(\omega)<1$ for each $\omega \in \Omega$, where $M(\omega)=\|T(\omega)(X)\|$.

\section{Random Integral Equations}

In this section we shall apply the results of previous section to nonlinear functional random integral equations involving the Caratheodory functions for proving the existence of the random solution.

Given a closed and bounded interval $J=[0,1]$ in $\boldsymbol{R}$, the set of all real numbers, consider the nonlinear functional random integral equation (in short RIE),

$$
\left.x(t, \omega)=q(t, \omega)+\int_{0}^{\sigma(t)} f(s, x(\eta(s), \omega)), \omega\right) d s, \quad t \in J,
$$

here $q: J \times \Omega \rightarrow \boldsymbol{R}, f: J \times \boldsymbol{R} \times \Omega \rightarrow R$ and $\sigma, \eta: J \rightarrow J$.

Let $C(J, \boldsymbol{R})$ and $B M(J, \boldsymbol{R})$ denote respectively the spaces of all continuous and bounded and measurable real-valued functions on $J$. We define a norm $\|\cdot\|_{C}$ in $C(J, \boldsymbol{R})$ by $\|x\|_{C}=\sup _{t \in J}|x(t)|$ and a norm $\|\cdot\|_{B}$ in $B M(J, \boldsymbol{R})$ by $\|x\|_{B}=\max _{t \in J}|x(t)|$. 
Obviously $C(J, \boldsymbol{R}) \subset B M(J, \boldsymbol{R})$. We shall seek the random solution of the RIE (3.1) in the space $B M(J, \boldsymbol{R})$ under suitable conditions. We need the following definition in the sequel.

Definition 3.1. A function $\beta: J \times \boldsymbol{R} \times \Omega \rightarrow \boldsymbol{R}$ is called $L_{\omega}^{1}$ Caratheodory if for each $\omega \in \Omega$,

(i) $t \mapsto f(t, x, \omega)$ is measurable for all $x \in \boldsymbol{R}$,

(ii) $x \mapsto f(t, x, \omega)$ is almost everywhere continuous for $t \in J$, and

(iii) for given real number $k>0$, there exists a function $h_{k}: \Omega \rightarrow L^{1}(J, \boldsymbol{R})$ such that

$$
|f(t, x, \omega)| \leq h_{k}(t, \omega), \quad \text { a.e. } t \in J
$$

for all $x \in \boldsymbol{R}$ with $|x| \leq k$.

We consider the following assumptions:

$\left(\mathbf{H}_{0}\right)$ The functions $\sigma, \eta: J \rightarrow J$ are continuous with $\sigma(t) \leq t$ and $\eta(t) \leq t$ for all $t \in J$

$\left(\mathbf{H}_{1}\right) q: \Omega \rightarrow C(J, \boldsymbol{R})$ is measurable,

$\left(\mathbf{H}_{2}\right) f(t, x, \omega)$ is $L_{\omega}^{1}$-Caratheodory,

$\left(\mathbf{H}_{3}\right) \omega \mapsto f(t, x, \omega)$ is measurable for all $t \in J$ and $x \in \boldsymbol{R}$.

$\left(\mathbf{H}_{4}\right)$ There exists a function $\phi: \Omega \rightarrow L^{1}(J, \boldsymbol{R})$ and a continuous nondecreasing function $\psi:[0, \infty) \rightarrow(0, \infty)$ such that

$$
|f(t, x, \omega)| \leq \phi(t, \omega) \psi(|x|), \quad \text { a.e. } t \in J
$$

for all $x \in \boldsymbol{R}$ and $\omega \in \Omega$.

Theorem 3.1. Suppose that the assumptions $\left(\mathbf{H}_{0}\right)-\left(\mathbf{H}_{4}\right)$ hold. Further if for each $\omega \in \Omega$,

$$
\int_{\|q(\omega)\|_{C}}^{\infty} \frac{d s}{\psi(s)}>\|\phi(\omega)\|_{L^{1}}
$$

then the RIE (3.1) has a random solution on $J$.

Proof. It is known that $B M(J, \boldsymbol{R})$ is a separable Banach space. Let $\omega \in \Omega$ be fixed. Define an operator $T: \Omega \times B M(J, \boldsymbol{R}) \rightarrow B M(J, \boldsymbol{R})$ by

$$
T(\omega) x(t)=q(t, \omega)+\int_{0}^{\sigma(t)} f(s, x(\eta(s), \omega), \omega) d s, \quad t \in J .
$$

By $\left(\mathbf{H}_{1}\right), q(t, \omega)$ is measurable in $\omega$ for all $t \in J$. Now $\int_{0}^{\sigma(t)} f(s, x(\eta(s), \omega), \omega) d s$ is the limit of a finite sum of measurable functions, so $\omega \mapsto \int_{0}^{\sigma(t)} f(s, x(\eta(s), \omega), \omega) d s$, is measurable. Again the sum of two measurable functions is again measurable, and therefore $T(\omega)$ defines a random operator $T: \Omega \times B M(J, \boldsymbol{R}) \rightarrow B M(J, \boldsymbol{R})$. We shall show that $T(\omega)$ satisfies the conditions (a) and (b) of Theorem 2.1.

Step I: First we shall show that the random operator $T(\omega)$ is completely continuous on $B M(J, \boldsymbol{R})$. Since $f(t, x, \omega)$ is $L^{1}$-Caratheodory, using the standard arguments and the 
dominated convergence theorem it is proved that $T(\omega)$ is continuous random operator on $B M(J, \boldsymbol{R})$. Now let $S \subset B M(J, \boldsymbol{R})$ be bounded set with bound $k$. Then for each $\omega \in \Omega$, by $\left(\mathbf{H}_{3}\right)$,

$$
\begin{aligned}
T(\omega) x(t) & =q(t, \omega)+\int_{0}^{\sigma(t)}|f(s, x(\eta(s), \omega), \omega)| d s \\
& \leq\|q(\omega)\|_{C}+\int_{0}^{\sigma(t)} h_{k}(s, \omega) d s \\
& \leq\|q(\omega)\|_{C}+\left\|h_{k}(\omega)\right\|_{L^{1}}
\end{aligned}
$$

for all $x \in S$, showing that $\{(T(\omega)(S)\}$ is a uniformly bounded set in $B M(J, \boldsymbol{R})$ for each $\omega \in \Omega$. Now let $t, \tau \in J$. Then for any $x \in S$,

$$
\begin{aligned}
|T(\omega) x(t)-T(\omega) x(\tau)| \leq & |q(t, \omega)-q(\tau, \omega)| \\
& +\left|\int_{0}^{\sigma(t)} f(s, x(\eta(s), \omega), \omega) d s-\int_{0}^{\sigma(t)} f(s, x(\eta(s), \omega), \omega) d s\right| \\
\leq & |q(t, \omega)-q(\tau, \omega)|+|p(t, \omega)-p(\tau, \omega)|
\end{aligned}
$$

for each $\omega \in \Omega$, where $p(t, \omega)=\int_{0}^{\sigma(t)} h_{k}(s, \omega) d s$.

Since $t \mapsto q(t, \omega)$ is continuous on compact interval $J$, it is uniformly continous. Also $t \mapsto p(t, \omega)$ is a uniformly continuous, it follows from $(3.3)$ that $\{T(\omega)(S)\}$ is compact set in $B M(J, \boldsymbol{R})$ by Aezela - Ascoli theorem, for each $\omega \in \Omega$. Therefore $T$ is a completely continuous random operator on $\Omega \times B M(J, \boldsymbol{R})$.

Step II: Now for any solution $x$ to $T(\omega) x=\alpha(\omega) x, \alpha(\omega)>1$, one has

$$
\begin{aligned}
|x(t, \omega)| & \leq|T(\omega) x(t, \omega)| \\
& \leq|q(t, \omega)|+\int_{0}^{\sigma(t)}|f(s, x(\eta(s), \omega), \omega)| d s \\
& \leq|q(t, \omega)|+\int_{0}^{t} \phi(s, \omega) \psi(|x(\eta(s), \omega)|) d s \\
& \leq\|q(\omega)\|+\int_{0}^{t} \phi(s, \omega) \psi(|x(\eta(s), \omega)|) d s
\end{aligned}
$$

for each $\omega \in \Omega$.

For a fixed $\omega \in \Omega$, define $\mu(t, \omega)=\sup _{\tau \in[0, t]}|x(\tau, \omega)|$. Since $x: \Omega \rightarrow B M(J, \boldsymbol{R})$, there exists a $t^{*} \in[0, t]$ such that $\mu(t, \omega)=\left|x\left(t^{*}, \omega\right)\right|$. Hence from the inequality (3.4),

$$
\begin{aligned}
\mu(t, \omega) & =\left|x\left(t^{*}, \omega\right)\right| \leq\|q(\omega)\|_{C}+\int_{0}^{\sigma\left(t^{*}\right)} \phi(s, \omega) \psi(|x(\eta(s), \omega)|) d s \\
& \leq\|q(\omega)\|_{C}+\int_{0}^{t} \phi(s, \omega) \psi(\mu(t, \omega)) d s
\end{aligned}
$$


Let $\mu(t, \omega)=\|q(\omega)\|_{C}+\int_{0}^{t} \phi(s, \omega) \psi(\mu(t, \omega)) d s$.

Then $\mu(t, \omega) \leq u(t, \omega)$, and

$$
\begin{aligned}
\frac{d u(t, \omega)}{d t} & =\phi(t, \omega) \psi(\mu(t, \omega)) \leq \phi(t, \omega) \psi(u(t, \omega)) \\
u(0, \omega) & =\|q(\omega)\|_{C}
\end{aligned}
$$

Hence we have

$$
\begin{aligned}
\frac{u^{\prime}(t, \omega)}{\psi(u(t, \omega))} & =\phi(t, \omega) \\
u(0, \omega) & =\|q(\omega)\|_{C} .
\end{aligned}
$$

Integrating over 0 to $t$,

$$
\int_{0}^{t} \frac{d u(t, \omega)}{\psi(u(t, \omega))} d t \leq \int_{0}^{t} \phi(s, \omega) d s \leq \int_{0}^{1} \phi(s, \omega) d s .
$$

By the change of the variable formula, we obtain

$$
\int_{\|q(\omega)\|_{C}}^{\mu(t, \omega)} \frac{d s}{\psi(s)} \leq\|\phi(\omega)\|_{L^{1}}<\int_{\|q(\omega)\|_{C}}^{\infty} \frac{d s}{\psi(s)} .
$$

The inequality shows that there exists a constant $K>0$ such that for each $\omega \in \Omega$, $u(t, \omega) \leq K$ for all $t \in J$ and hence $\mu(t, \omega) \leq K$ for all $t \in J$. Since $|x(t, \omega)| \leq \mu(t, \omega)$ for every $t \in J$, we have $\|x(\omega)\|_{B} \leq k$ for each $\omega \in \Omega$. Thus the condition (b) of Theorem 2.1 is satisfied. Now an application of Corollary 2.1 yields that the RIE (3.1) has a random solution on $J$. This completes the proof.

As an application of Theorem 3.1, we consider the nonlinear functional random differential equation (in short RDE)

$$
\left.\begin{array}{r}
\frac{d u(t, \omega)}{d t}=f(t, x(\eta(t), \omega), \omega) \quad \text { a.e. } t \in J \\
x(0, \omega)=q(\omega)
\end{array}\right\}
$$

where $q: \Omega \rightarrow \boldsymbol{R}$ is a real-valued random variable, $f: J \times R \times \Omega \rightarrow \boldsymbol{R}$ and $\eta: J \rightarrow J$ is continuous.

By the random solution to the RDE (3.6), we mean a measurable function $x: \Omega \rightarrow$ $A C(J, \boldsymbol{R})$ that satisfies the equations (3.6), there $A C(J, \boldsymbol{R})$ is a space of all absolutely continuous real-valued functions on $J$.

Theorem 3.2. Assume that the hypotheses $\left(\mathbf{H}_{2}\right),\left(\mathbf{H}_{4}\right)$ hold. Further if $\eta(t) \leq t$ for all $t \in J$ and if for each $\omega \in \Omega$,

$$
\int_{\|q(\omega)\|_{C}}^{\infty} \frac{d s}{\psi(s)}>\|\phi(\omega)\|_{L^{1}}
$$


holds, then the RDE (3.6) has a random solution on $J$.

Proof. The RDE (3.6) is equivalent to the random integral equation

$$
x(t, \omega)=q(\omega)+\int_{0}^{t} f(s, x(\eta(s), \omega), \omega) d s, \quad t \in J .
$$

Now the desired conclusion follows by an application of Theorem 3.1 with $q(t, \omega)=$ $q(\omega)$ and $\sigma(t)=t$ for all $t \in J$. In this case $A C(J, \boldsymbol{R}) \subset B M(J, \boldsymbol{R})$. This completes the proof.

\section{References}

[1] J. Banas and K. Goebel, Measures of Noncompactness in Banach Spaces, Marcel Dekker Inc. New York, 1980.

[2] A. T. Bharucha Reid, Random Integral Equations, Academic Press, New York, 1972.

[3] B. C. Dhage, On $\alpha$-condensing mappings in Banach algebras, The Math. Student 64(1994), 146-152.

[4] B. C. Dhage, Random fixed point theorems in Banach algebras with applications to nonlinear random integral equations, Tamkang J. Math. 34(2003), 29-43.

[5] C. J. Himmelberg, Measurable relations, Fund. Math. 87(1975), 53-72.

[6] M. A. Kransoselskii, Topological Methods in the Theory of Nonlinear Integral Equations, Pregamon Press, 1964.

[7] S. Itoh, Random fixed point theorems with an applications the random differential equations in Banach spaces, J. Math. Anal. Appl. 67(1979), 261-273.

[8] K. Kuratowskii and C. Ryll-Nardzewski, A general theorem on selectors, Bull. Acad. Polons, Sci. Ser. Math. Sci. Astr. Phys. 13(1965), 397-403.

[9] M. Martelli, A Rothe's theorem for noncompact acyclic-valued map, Bull. Un. Mat. Ital. 4(1975), 70-76.

[10] H. Schaefer, Uber die method der a priori-schranken, Math. Ann. 126(1955), 415-416.

Gurukul Colony, Ahmedpur - 413 515, Dist. Latur, Maharashtra, India.

E-mail: bcd20012001@yahoo.co.in 